\title{
Metabolism of Black Carrot Polyphenols during In Vitro Fermentation Is Not Affected by Cellulose or Cell Wall Association
}

\author{
Gabriele Netzel ${ }^{1}$, Deirdre Mikkelsen ${ }^{1,2, *}$, Bernadine M. Flanagan ${ }^{1}$, Michael E. Netzel ${ }^{1}$, \\ Michael J. Gidley ${ }^{1}$ and Barbara A. Williams ${ }^{1}$ \\ 1 Centre for Nutrition and Food Sciences, Queensland Alliance for Agriculture and Food Innovation, \\ The University of Queensland-St. Lucia Campus, Brisbane, QLD 4072, Australia; g.netzel@uq.edu.au (G.N.); \\ b.flanagan@uq.edu.au (B.M.F.); m.netzel@uq.edu.au (M.E.N.); m.gidley@uq.edu.au (M.J.G.); \\ b.williams@uq.edu.au (B.A.W.) \\ 2 School of Agriculture and Food Sciences, The University of Queensland-St. Lucia Campus, \\ Brisbane, QLD 4072, Australia \\ * Correspondence: d.mikkelsen@uq.edu.au; Tel.: +61-7-3346-9642
}

Received: 26 October 2020; Accepted: 12 December 2020; Published: 21 December 2020

check for updates

\begin{abstract}
Fruit and vegetable polyphenols are associated with health benefits, and those not absorbed could be fermented by the gastro-intestinal tract microbiota. Many fermentation studies focus on "pure" polyphenols, rather than those associated with plant cell walls (PCW). Black carrots $(\mathrm{BlkC})$, are an ideal model plant food as their polyphenols bind to PCW with minimal release after gastro-intestinal digestion. BlkC were fractionated into three components-supernatant, pellet after centrifugation, and whole puree. Bacterial cellulose (BCell) was soaked in supernatant (BCell\&S) as a model substrate. All substrates were fermented in vitro with a pig faecal inoculum. Gas kinetics, short chain fatty acids, and ammonium production, and changes in anthocyanins and phenolic acids were compared. This study showed that metabolism of BlkC polyphenols during in vitro fermentation was not affected by cellulose/cell wall association. In addition, BCell\&S is an appropriate model to represent BlkC fermentation, suggesting the potential to examine fermentability of PCW-associated polyphenols in other fruits/vegetables.
\end{abstract}

Keywords: bacterial cellulose; in vitro fermentation; black carrot; polyphenols; anthocyanins; phenolic acids

\section{Highlights}

- $\quad$ Black carrot fractions (puree, supernatant, and pellet) differed in fermentability

- $\quad$ Polyphenol fermentations were comparable between carrots and a cellulose model

- Juice-soaked cellulose can be a model for vegetable polyphenol fermentation

\section{Introduction}

Anthocyanins and phenolic acids are considered to be important components of the health properties of fruits and vegetables [1-4]. From epidemiological studies and associated meta-analyses in the last two decades, as well as more recent in vivo studies, evidence is accumulating to show that such polyphenol-containing plant foods, when present in the diet, may exert significant health benefits in humans. These include associations with a reduction in the incidence of diabetes, cardiovascular disease, some cancers, and inflammation [2,5-9]. In unprocessed plants, such as fruits or vegetables, polyphenols are present within the plant cell (PC) vacuole, attached to the plant cell wall (PCW), or both $[10,11]$. This leads to questions concerning their bioaccessibility and subsequent bioavailability, 
particularly for those compounds which are ingested as part of a plant food, rather than as a purified compound [12].

Bioaccessibility is defined as the amount of an ingested nutrient/dietary compound that is available for absorption in the gastro-intestinal tract after digestion in the small intestine [13,14]. For foods of plant origin, this can be affected by factors such as configuration of the PCW, location within the PCW, and interactions with PCW components [11,12,15-20]. Bioavailability, on the other hand, describes the actual amounts of nutrients/dietary compounds which are absorbed from the gastrointestinal tract (GIT) into the bloodstream [18,21]. Polyphenols which are not directly absorbed may subsequently be metabolised by the GIT microbiota [2,22,23], usually in the large intestine. Consequently, there is also growing interest in the properties of their fermentative metabolites, including their potential absorption, interactions with the enterocyte, modulatory effects on the GIT microbiota (stimulating the growth of specific beneficial bacteria thus exhibiting prebiotic properties), and inhibitory effects on inflammatory processes known to be associated with disease states [23-25].

Fermentation of a range of different "pure" polyphenols has been investigated, including various anthocyanins and phenolic acids [21,23,26-31]. The consequence of in vitro binding of single pure polyphenols to cellulose or isolated cell walls has also recently been reported [32].

Usually, methods for extraction of PCW are harsh, and the resultant product may no longer represent the in planta structure, particularly in relation to delicate interactions between polyphenols and the PCW. The bacterium Komagataeibacter xylinus is used as a cellulose biosynthesis model, since it has the same general features of cellulose deposition as found in plants [33]. One advantage of this PCW analogue approach is to avoid destruction of polyphenols (such as by oxidation), as might occur otherwise $[11,17,33]$.

Of the many fruits and vegetable consumed for their high antioxidant properties, black carrots (Daucus carota sp. sativus var. atrorubens) contain both dietary fibre and polyphenol compounds, such as acylated and non-acylated anthocyanins and phenolic acids [34-38]. Additionally, black carrots are a cheap and substantial source of polyphenols which may be used to enrich food products [39]. It is also known that black carrot anthocyanins and phenolic acids bind to PCW with minimal release after gastric and small intestinal in vitro digestion [40], thus reaching the large intestine intact. To date, digestibility studies have not examined fermentation of non-bioaccessible polyphenols by large intestinal bacteria, which is also required to elucidate the relation between PCW and attached polyphenols in the entire GIT.

In this study, blanched black carrots were fractionated, and the fermentability compared with bacterial cellulose (BCell), both as is, and combined with, carrot juice. The aims were (i) to compare the fermentability of carrot PCW with purified BCell; (ii) to determine whether the carrot PCW versus BCell would make a difference to polyphenolic bioaccessibility; and (iii) to ferment BCell combined with black carrot juice to determine whether such a model of carrot polyphenols attached to pure cellulose, would be an appropriate system to represent the fermentability of black carrots. If so, such a construction could be suitable to examine fermentation of polyphenols and other phytochemicals associated with fruits or vegetables, more generally.

\section{Materials and Methods}

\subsection{Materials}

All solvents were of HPLC grade (Merck Serono Australia Pty Ltd., Kilsyth, VIC, Australia), cy-3-O-glucoside was purchased from ChromaDex (Irvine, CA, USA). Phenolic acid standards were obtained from Sigma-Aldrich (Castle Hill, NSW, Australia). If not otherwise stated, all chemicals and standards were purchased from Merck or Sigma-Aldrich. 


\subsection{Preparation of Substrates}

Black carrots ( $2 \mathrm{~kg}$ ) were purchased from a local supermarket (Brisbane, QLD, Australia), washed thoroughly and manually cut into $1 \mathrm{~cm}$ pieces. The pieces were then blanched at $90{ }^{\circ} \mathrm{C}$ in an equal amount (by weight) of water for $3 \mathrm{~min}$, and drained and cooled to $5{ }^{\circ} \mathrm{C}$. A puree was prepared by blending the pieces in an equal amount of blanching water for 2 min using a domestic blender (BJB840XL, $1800 \mathrm{~W}$, Breville, Botany, NSW, Australia). The puree was then separated into two parts. One part was kept as whole puree (BlkC_WP), the other part was separated into pellet (BlkC_P) and supernatant (BlkC_S) by centrifugation at $18,900 \times \mathrm{g}$ for $15 \mathrm{~min}$ at $10{ }^{\circ} \mathrm{C}$ (Beckman Coulter, Brea, CA, USA). An aliquot $(600 \mathrm{~mL})$ of the supernatant was kept aside for BCell soaking. Following blanching, all procedures were conducted under cool conditions $\left(10^{\circ} \mathrm{C}\right)$.

BlkC_WP, BlkC_P, and the remaining BlkC_S fractions were freeze-dried and cryo-ground (6850 SPEX Freezer/Mill; Metuchen, NJ, USA), using pre-cooling for $5 \mathrm{~min}$, followed by two cycles of grinding at an impactor speed of 10/s for 5 min each, with an intermediate cooling time of 2 min between cycles. All dried black carrot substrates were stored at room temperature in a desiccator pending further use.

Bacterial cellulose was produced as described previously [33]. Briefly, Komagataeibacter xylinus strain ATCC 53524 (Cryosite, South Granville, NSW, Australia) was cultivated in HS medium at pH 5.0 [41]. After $72 \mathrm{~h}$ incubation at $30^{\circ} \mathrm{C}, \mathrm{BCell}$ pellicles were harvested and washed in ice-cold sterile Milli-Q water with gentle agitation and frequent rinsing to remove excess medium and bacterial cells. Once purified, the BCell was divided into two portions: (i) pure BCell, and (ii) BCell\&S (BCell which was soaked in black carrot supernatant for $12 \mathrm{~h}$, rinsed thoroughly with ice-cold sterile Milli-Q water for $2 \mathrm{~h}$ with gentle agitation (100 rpm), to remove unbound polyphenols and sugars). The ratio of BCell to black carrot supernatant (juice) was: $1.96 \mathrm{~g}$ (dry weight) of $\mathrm{BCell}$ in $500 \mathrm{~mL}$ of juice. The $\mathrm{pH}$ of the juice was 6.2. All cellulosic materials were freeze-dried, cryo-ground and then stored as described above.

\subsection{Collection and Preparation of Inoculum (Faecal)}

Faecal collection was according to the method of Williams et al. (Williams et al., 2005b). In brief, faeces was collected from five Large White pigs (30-35 kg) fed a semi-purified diet for at least ten days. This diet was based on highly digestible corn starch and fishmeal, and had been formulated to be as free as possible of potentially fermentable carbohydrates, to avoid microbial adaptation to any of the substrates being tested. The pigs were not exposed to antibiotics. All procedures involving the pigs had been approved by the University of Queensland Animal Ethics Committee (NRAVS/244/09/CSIRO).

Following collection, fresh faeces were placed immediately into pre-warmed vacuum flasks pre-flushed with $\mathrm{CO}_{2}$, prior to transport to the laboratory. At the laboratory, faeces were mixed with pre-warmed $\left(39^{\circ} \mathrm{C}\right)$, anaerobic, sterile saline $\left(0.9 \mathrm{~g} \mathrm{~L}^{-1} \mathrm{NaCl}\right)$. The ratio between faeces and saline (1:5) was based on the consistency of the faeces, to allow injection into serum bottles. This diluted mixture was homogenised using a hand-mixer for $60 \mathrm{~s}$ and strained through four layers of muslin cloth to remove particulates. All procedures were conducted under a constant flow of $\mathrm{CO}_{2}$ to maintain a strictly anaerobic environment.

\subsection{Cumulative Gas Production Technique}

Gas production was measured according to the in vitro method described previously [42]. In brief: approximately $0.12 \mathrm{~g}$ of each substrate was weighed into replicate $60 \mathrm{~mL}$ serum bottles. Two $\mathrm{mL}$ of inoculum was added to $38 \mathrm{~mL}$ of a semi-purified medium in each bottle, within $2 \mathrm{~h}$ of collection. Following inoculation, the bottles were positioned randomly in a pre-warmed incubator at $39^{\circ} \mathrm{C}$. Duplicate bottles for time point intervals at $0,4,8,12$, and $24 \mathrm{~h}$ were removed, while quadruplicate bottles were fermented for $48 \mathrm{~h}$ for end-point analyses. Upon removal, all bottles were immediately placed into an ice-slurry water-bath for $15 \mathrm{~min}$, to inhibit bacterial activity and thereby stop fermentation. Bottles to be analysed for anthocyanins and phenolic acids were then frozen pending sampling. Later, 
they were thawed and $1 \mathrm{~mL}$ aliquots collected from all bottles (including the $48 \mathrm{~h}$ ) and acidified with formic acid (1:1.1) for anthocyanin and phenolic acid analyses. The acidified aliquots were stored at $-20{ }^{\circ} \mathrm{C}$ until further analysis. One "Blank" (inoculum and medium only) was included, which was not included in the analysis of variance, but the results are shown in the appropriate tables.

\subsection{Short Chain Fatty Acid (SCFA) and Ammonium Analyses}

Short chain fatty acid analysis was conducted according to a previously described method [43]. Samples were thawed, prepared by vacuum distillation, and analysed by gas chromatography (Agilent 6890 Series GC, Agilent Technologies, Wilmington, DE, USA), using a fused silica column (J\&W Scientific, supplied by Agilent) with a $1 \mu \mathrm{m}$ coating. Helium was the carrier gas (flow rate of $6 \mathrm{~mL} / \mathrm{min}$ ). Split injector and FID detector were held at $250{ }^{\circ} \mathrm{C}$. The oven was held at $90{ }^{\circ} \mathrm{C}$ for $1 \mathrm{~min}$, then ramped to $190^{\circ} \mathrm{C}$ (rate of $10^{\circ} \mathrm{C} / \mathrm{min}$ ) and held for $1 \mathrm{~min}$. SCFA values were corrected to mmoles per gram DM weighed into the serum bottles prior to inoculation.

Ammonium analysis was carried out using a modified colorimetric method [44]. The method is based on the chemical reaction of $\mathrm{NH}_{4}{ }^{+}$ions with sodium salicylate and nitroprusside in a weakly alkaline buffer, at a wavelength of $650 \mathrm{~nm}$, using a UV/visible spectrophotometer (Automated Discrete Analyser Model AQ2+, SEAL Analytical Ltd., Fareham, UK).

\subsection{Sugar Analysis}

Samples were quantitatively analysed for sugars using ${ }^{1} \mathrm{H}$ NMR in a similar way to a published method [45]. Briefly, $20 \mathrm{mg}$ of dry, ground material was suspended or dissolved at $80{ }^{\circ} \mathrm{C}$ overnight in $650 \mu \mathrm{L} \mathrm{D} \mathrm{D}_{2} \mathrm{O}$. When the samples were cooled to room temperature, $100 \mu \mathrm{L}$ of Sodium 3-(Trimethylsilyl)propionate-2,2,3,3-d4 (TSP) in $\mathrm{D}_{2} \mathrm{O}(12 \mathrm{mg} / \mathrm{mL})$ was added as an internal standard and samples were filtered into $5 \mathrm{~mm}$ NMR tubes. NMR spectra were measured on a Bruker Avance $500 \mathrm{MHz}$ spectrometer (Bruker, Billerica, MA, USA) operating at $298 \mathrm{~K}$ equipped with a $5 \mathrm{~mm}$ PABBO probe using a $12 \mu \mathrm{s} 90^{\circ}$ pulse, $3.91 \mathrm{~s}$ acquisition time, a $20 \mathrm{~s}$ relaxation delay and $64 \mathrm{scans}$. All samples were run a minimum of four times for verification.

\subsection{Analysis of Anthocyanins and Phenolic Acids}

Anthocyanins (cy-3-O-xylglcgal, cy-3-O-xylgal, caffeic acid derivative of cy-3-O-xylglcgal, sinapic acid derivative of cy-3-O-xylglcgal, ferulic acid derivative of cy-3-O-xylglcgal, and p-coumaric acid derivative of cy-3-O-xylglcgal) and phenolic acids (3-O-caffeoylquinic (chlorogenic), 5-O-caffeoylquinic (neochlorogenic), caffeic, sinapic, ferulic and p-coumaric acid) were determined by HPLC-PDA and ESI-LC/MS as described previously [11,17,32].

\subsection{Data Handling and Statistical Analysis}

All gas, SCFA and ammonium data were converted to units per gram DM of weighed-in substrate. Anthocyanins, phenolic acids, and metabolites are expressed as nmol per gram DM weighed-in substrate.

Gas profiles of cumulative gas were fitted to the monophasic model of Groot et al. [46]:

$$
\mathrm{DMCV}=\frac{\mathrm{A}}{1+(\mathrm{C} / \mathrm{t})^{\mathrm{B}}}
$$

where $\mathrm{DMCV}=$ cumulative gas produced at time $\mathrm{t}(\mathrm{mL}) ; \mathrm{A}=$ asymptotic gas production; $\mathrm{B}=$ switching characteristic of the curve; $\mathrm{C}=$ time $(\mathrm{h})$ at which half of the asymptotic value has been reached-also $\mathrm{T} \frac{1}{2}$; and $\mathrm{t}=$ time $(\mathrm{h})$. 
The maximum rate of gas production $\left(\mathrm{R}_{\mathrm{Max}}\right)$, and the time at which it occurs $\left(\mathrm{T}_{\mathrm{RMax}}\right)$, were calculated using A, B and C values from Equation (1), according to the following equations [47]:

$$
\begin{gathered}
\mathrm{T}_{R \max }=\mathrm{C} \times\left(((\mathrm{B}-1) /(\mathrm{B}+1))^{(1 / \mathrm{B})}\right) \\
\mathrm{R}_{\operatorname{Max}}=\left(\mathrm{A} \times\left(\mathrm{C}^{\mathrm{B}}\right) \mathrm{B}\left(\mathrm{T}_{\mathrm{Rmax}}{ }^{(-\mathrm{B}-1)}\right)\right) /\left(1+\left(\mathrm{C}^{\mathrm{B}}\right) \mathrm{T}_{\mathrm{R} \max }{ }^{(-\mathrm{B})}\right)^{2}
\end{gathered}
$$

Differences between substrates were tested for significance using Tukey's studentized range test of multiple comparisons according to

$$
Y=\mu+S_{i}+\varepsilon_{i}
$$

where $\mathrm{Y}$ is the result; $\mu$ is the mean; $\mathrm{S}_{\mathrm{i}}$ is the effect of differences between substrates; and $\varepsilon_{i}$ is the error term. The effect of replicate was tested separately, but was not significant $(p>0.05)$ for any of the parameters, and so was removed for the final analysis. Values for blanks (medium and inoculum) were also excluded.

All statistical analyses were performed using the SAS NLIN version 9.1 (curve-fitting) and GLM (significant differences) procedures (Statistical Analysis Systems Institute Inc., Cary, NC, USA).

\section{Results and Discussion}

Percentage dry matter was determined for the substrates prior to fermentation and was as follows: BCell-94.3\%; BCell\&S-85.5\%; BlkC_WP-94.2\%; BlkC_S-85.5\%; BlkC_P-94.2\%; and BlkC_S-87.4\%. These values were used to correct all analytical results to /gDM. Table 1 shows a summary of the simple sugar analysis of the substrates.

Table 1. Percentage water extractable sugars $w / w$ dry matter (mean \pm SE) for bacterial cellulose (BCell) and with added black carrot (BlkC) supernatant, and the BlkC fractions (whole puree (WP), supernatant (S), and pellet (P)).

\begin{tabular}{ccccc}
\hline Substrates $^{*}$ & \% Sucrose & \% Glucose & \% Fructose & \% Total Sugars ** \\
\hline BCell\&S & $7.3 \pm 0.6$ & $5.9 \pm 0.1$ & $3.7 \pm 0.2$ & $16.8 \pm 0.9$ \\
\hline BlkC_WP & $39.8 \pm 3.1$ & $3.4 \pm 0.3$ & $20.5 \pm 0.7$ & $63.6 \pm 4.1$ \\
\hline BlkC_S & $59.8 \pm 3.2$ & $5.7 \pm 0.3$ & $31.9 \pm 1.9$ & $97.4 \pm 5.4$ \\
\hline BlkC_P & $10.8 \pm 0.6$ & $1.2 \pm 0.1$ & $5.9 \pm 0.1$ & $17.8 \pm 0.8$
\end{tabular}

* BCell alone was not determined, as it is known to have no soluble sugars. ${ }^{* *} \%$ Total Sugars is the sum of sucrose, glucose and fructose.

\subsection{Concentration of Anthocyanins and Phenolic Acids in Pre-Fermented Substrates}

Table 2 shows the concentrations of acylated and non-acylated anthocyanins as well as phenolic acids in the black carrot substrates prior to fermentation.

Total anthocyanins (sum of acylated and non-acylated pigments) were highest in BlkC_S, followed by BCell\&S, BlkC_P, and BlkC_WP respectively. A similar distribution pattern was observed for phenolic acids with the highest concentration in BlkC_S, followed by BlkC_WP, BCell\&S, and BlkC_P. The acylated and non-acylated anthocyanin profiles found in the experimental black carrot substrates were similar to those reported by others [35,36]. In accordance with a previous report [48], chlorogenic acid (5-caffeoylquinic acid) could be detected as the predominant phenolic acid in all substrates whereas neochlorogenic acid (3-caffeoylquinic acid) was found in much lower concentrations. In addition to the caffeoylquinic acids, four non-esterified hydroxycinnamic acids could be identified and quantified in all substrates: caffeic, ferulic, p-coumaric and sinapic acid. Caffeic and ferulic acid have been reported to occur as free phenolic acids in coloured carrots [48,49], whereas p-coumaric and sinapic acid most likely originated from their corresponding acylated anthocyanins. However, the total amount of these free hydroxycinnamic acids was less than $7.5 \%$ of total phenolic acids. It is also important to note that, given the wide variation in DM\% values, all anthocyanin and phenolic acid values are reported as per gram DM. 
Table 2. Anthocyanin and phenolic acid concentrations in the substrates (nmol/g DM) prior to fermentation.

\begin{tabular}{|c|c|c|c|c|}
\hline & BlkC_WP & BlkC_S & BlkC_P & BCell\&S \\
\hline \multicolumn{5}{|c|}{ Anthocyanins } \\
\hline Cy-3-O-xylglcgal & $319.4^{*}$ & 1584 & 655.0 & 587.3 \\
\hline Cy-3-O-xylgal & 1109 & 5568 & 2660 & 2118 \\
\hline Sum (non-acylated) & 1428 & 7152 & 3315 & 2705 \\
\hline Caffeic acid derivative of cy-3-O-xylglcgal & 96.5 & 129.0 & 70.9 & 66.5 \\
\hline Sinapic acid derivative of cy-3-O-xylglcgal & 479.6 & 948.6 & 471.8 & 633.8 \\
\hline Ferulic acid derivative of cy-3-O-xylglcgal & 5859 & 13380 & 6259. & 8059 \\
\hline p-coumaric acid derivative of cy-3-O-xylglcgal & 663.0 & 1496 & 778.3 & 860.6 \\
\hline Sum (acylated) & 7098 & 15,954 & 7580 & 9620 \\
\hline Total (acylated + non-acylated) & 8526 & 23,106 & 10,895 & 12,325 \\
\hline \multicolumn{5}{|c|}{ Phenolic acids } \\
\hline Chlorogenic acids & 27009 & 51052 & 16075 & 25597 \\
\hline Non-esterified hydroxycinnamates & 1832 & 3283 & 1271 & 1720 \\
\hline Total & 28,841 & 54,336 & 17,346 & 27,317 \\
\hline Dry matter $(\%)$ & 6.7 & 3.8 & 12.3 & 31.5 \\
\hline
\end{tabular}

* Data are arithmetic means, $n=3(\mathrm{CV} \leq 5 \%)$; Chlorogenic acids: sum of 5-caffeoylquinic acid and 3-caffeoylquinic acid; Non-esterified hydroxycinnamates: sum of caffeic, sinapic, ferulic and p-coumaric acid. 


\subsection{Fermentability of Black Carrot vs. BCell Model System}

Tables 3 and 4, and Figure 1, show results for in vitro fermentation parameters, both in terms of kinetics and end-products ( $48 \mathrm{~h})$.

Table 3. Cumulative gas kinetics and end-point parameters for bacterial cellulose alone (BCell) and with added BlkC supernatant (BCell\&S), and black carrot (BlkC) fractions (whole puree (WP), supernatant (S), and pellet $(\mathrm{P}))$.

\begin{tabular}{cccccccc}
\hline Substrates & $\mathbf{n}$ & $\mathbf{D M C V}(\mathbf{m L})$ & $\frac{1}{2}$ Time $(\mathbf{h})$ & $\mathbf{T R}_{\max }(\mathbf{h})$ & $\mathbf{R}_{\max }(\mathbf{L m} / \mathbf{h})$ & $\mathbf{p H}$ & $\mathbf{N H}_{\mathbf{4}}$ mmol/bottle \\
\hline BCell & 3 & $111^{\mathrm{c}}$ & $23.97^{\mathrm{b}}$ & $12.09^{\mathrm{b}}$ & $0.413^{\mathrm{d}}$ & $6.50^{\mathrm{a}}$ & $2.50^{\mathrm{a}}$ \\
BCell\&S & 4 & $331^{\mathrm{b}}$ & $18.04^{\mathrm{c}}$ & $12.35^{\mathrm{b}}$ & $1.455^{\mathrm{b}}$ & $6.35^{\mathrm{b}}$ & $2.22^{\mathrm{a}}$ \\
BlkC_WP & 4 & $377^{\mathrm{a}}$ & $23.10^{\mathrm{b}}$ & $12.96^{\mathrm{b}}$ & $1.445^{\mathrm{b}}$ & $6.37^{\mathrm{ab}}$ & $1.16^{\mathrm{a}}$ \\
BlkC_S & 4 & $396^{\mathrm{a}}$ & $15.3^{\mathrm{c}}$ & $9.23^{\mathrm{b}}$ & $1.965^{\mathrm{a}}$ & $6.34^{\mathrm{b}}$ & $2.61^{\mathrm{a}}$ \\
BlkC_P & 4 & $394^{\mathrm{a}}$ & $31.9^{\mathrm{a}}$ & $18.43^{\mathrm{a}}$ & $1.205^{\mathrm{c}}$ & $6.37^{\mathrm{ab}}$ & $2.00^{\mathrm{a}}$ \\
\hline Probability & & $<0.0001$ & $<0.0001$ & $<0.0001$ & $<0.0001$ & 0.023 & 0.58 \\
MSD & 32.3 & 2.84 & 3.76 & 0.165 & 0.138 & 1.34 \\
\hline Blank & 2 & 8.7 & 20.3 & 10.29 & 0.310 & 6.47 & 0.30 \\
\hline
\end{tabular}

n: number of replicates analysed, DMCV: cumulative volume per gram DM weighed in (ml), $T \frac{1}{2}$ : value for " $\mathrm{C}$ " from curve-fitting, MSD: minimum significant difference, $\mathrm{TR}_{\max }$ : time of maximum rate of gas production (h), $\mathrm{R}_{\max }$ : maximum rate of gas production $(\mathrm{ml} / \mathrm{h})$. Blank: medium + inoculum $^{\mathrm{a}, \mathrm{b}, \mathrm{c}, \mathrm{d}}$ Superscripts differing in the same column, indicate significant differences $(P<0.05)$.

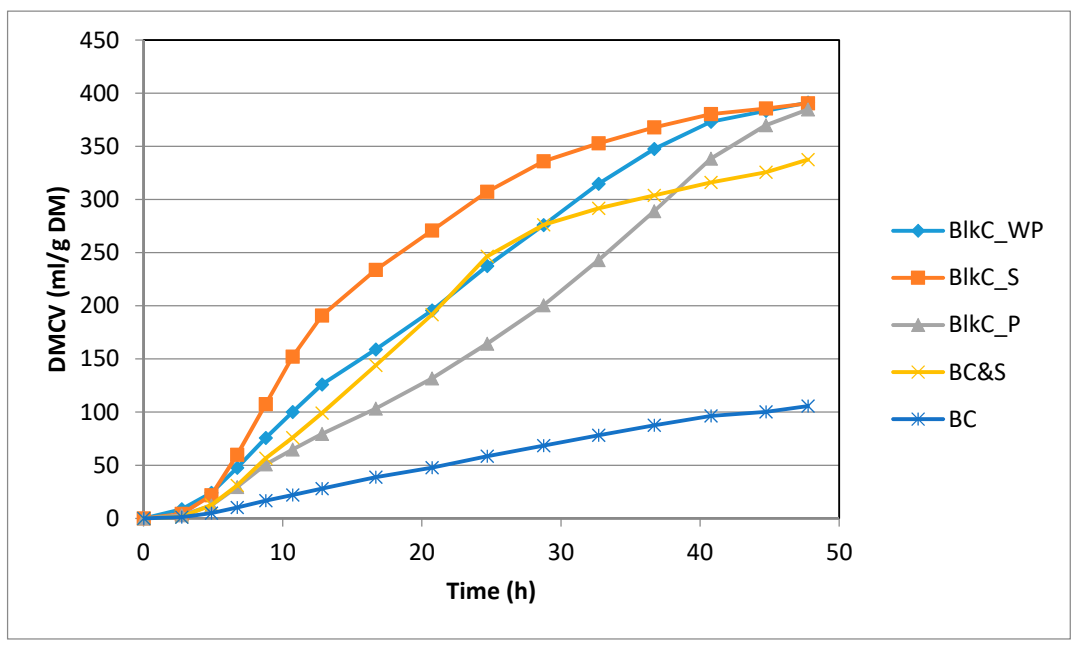

Figure 1. Representative gas profiles for the substrates Black Carrot-whole puree (BlkC_WP), supernatant (BlkC_S), pellet (BlkC_P) and Bacterial Cellulose, alone (BCell) and soaked in black carrot supernatant (BCell\&S).

In terms of fermentation kinetics and end-products (shown in Tables 3 and 4, and Figure 1), there was no significant difference in terms of fermentation end-products, including total gas and SCFA between the three components of black carrots. There were some differences, however, in the kinetics of fermentation with BlkC_S being the most rapidly fermented, with an earlier $\frac{1}{2}$ Time and faster $R_{\max }$. Blk-P was slowest and BlkC_WP was intermediate.

This is likely related to the simple sugar data (Table 1), as it can be seen that the supernatant contained about $97 \%$ simple sugars, followed by the whole puree and the pellet, comprising $63 \%$ and $18 \%$ simple sugars respectively. The model BCell\&S (soaked in supernatant) was most comparable with the BlkC_P in terms of total sugars, though had less end-product, and faster gas kinetics. BCell, was consistently the least well fermented substrate, having the slowest kinetics, and the least end-products. The presence of almost $17 \%$ simple sugars in the BCell\&S most likely played a role in the increased fermentation of this substrate compared to BCell. The released non-sugar components from BCell\&S (acylated and non-acetylated anthocyanins) may also have been a source of energy for the bacteria. 
Table 4. Fermentation end-products at $48 \mathrm{~h}$ for bacterial cellulose (BCell), and black carrots (BlkC) and their fractions (whole puree (WP), supernatant (S), and pellet (P)).

\begin{tabular}{|c|c|c|c|c|c|c|c|c|c|}
\hline \multirow{2}{*}{ Substrates } & \multirow{2}{*}{$\mathbf{n}$} & Acetic & Propionic & Butyric & Total SCFA & \%Acet & \%Prop & $\%$ But & BCR \\
\hline & & \multicolumn{4}{|c|}{$\mathrm{mmol} / \mathrm{gDM}$} & & & & \\
\hline BCell & 3 & $2.60^{b}$ & $0.83^{\mathrm{d}}$ & $0.32^{c}$ & $4.6^{c}$ & $56.4^{b}$ & $18.0^{\mathrm{e}}$ & $7.03^{a}$ & $0.452^{a}$ \\
\hline BCell\&S & 3 & $4.71^{\mathrm{a}}$ & $2.07^{\mathrm{c}}$ & $0.40^{b c}$ & $7.79^{b}$ & $60.5^{\mathrm{a}}$ & $26.6^{c}$ & $5.18^{c}$ & $0.165^{b}$ \\
\hline BlkC_WP & 3 & $5.79^{a}$ & $3.33^{a b}$ & $0.62^{a b}$ & $10.4^{\mathrm{ab}}$ & $55.5^{\mathrm{b}}$ & $31.9^{b}$ & $5.94^{\mathrm{b}}$ & $0.137^{\mathrm{b}}$ \\
\hline BlkC_S & 3 & $5.63^{\mathrm{a}}$ & $4.22^{\mathrm{a}}$ & $0.67^{\mathrm{a}}$ & $11.3^{\mathrm{a}}$ & $49.9^{c}$ & $37.4^{\mathrm{a}}$ & $5.90^{b}$ & $0.139^{b}$ \\
\hline BlkC_P & 3 & $6.17^{\mathrm{a}}$ & $2.42^{b c}$ & $0.70^{\mathrm{a}}$ & $9.9^{a b}$ & $62.1^{a}$ & $24.4^{\mathrm{d}}$ & $7.04^{\mathrm{a}}$ & $0.138^{b}$ \\
\hline Probability & & $<0.0001$ & $<0.0001$ & 0.0006 & 0.0002 & $<0.0001$ & $<0.0001$ & $<0.0001$ & $<0.0001$ \\
\hline MSD * & & 1.49 & 1.06 & 0.22 & 3.01 & 1.77 & 1.26 & 0.61 & 0.032 \\
\hline
\end{tabular}

n: number of replicates analysed, Acet: acetate, Prop: propionate, But: butyrate, BCR: branched chain ratio, ${ }^{*}$ MSD: minimum significant difference ${ }^{\text {a,b,c,d }}$ Superscripts differing in the same column, indicate significant differences $(P<0.05)$. 
Overall, the fermentability of the black carrot substrates followed expectations in terms of available energy in the form of carbohydrates. The substrate with the highest sugars, was the most fermentable, while the substrate with the highest cellulose content (BlkC_P) was least fermentable.

\subsection{Polyphenol Metabolism/Degradation during Fermentation}

\subsubsection{Anthocyanins}

The time-concentration degradation profiles of acylated and non-acylated anthocyanins, as well as total anthocyanins are shown for all substrates in Figure 2. Figure 2A shows the total anthocyanin content of each substrate as a function of fermentation time. An apparent lag phase until $12 \mathrm{~h}$ is observed followed by a decline to a very low level after $48 \mathrm{~h}$. However, individual sub-families of anthocyanins may behave differently.

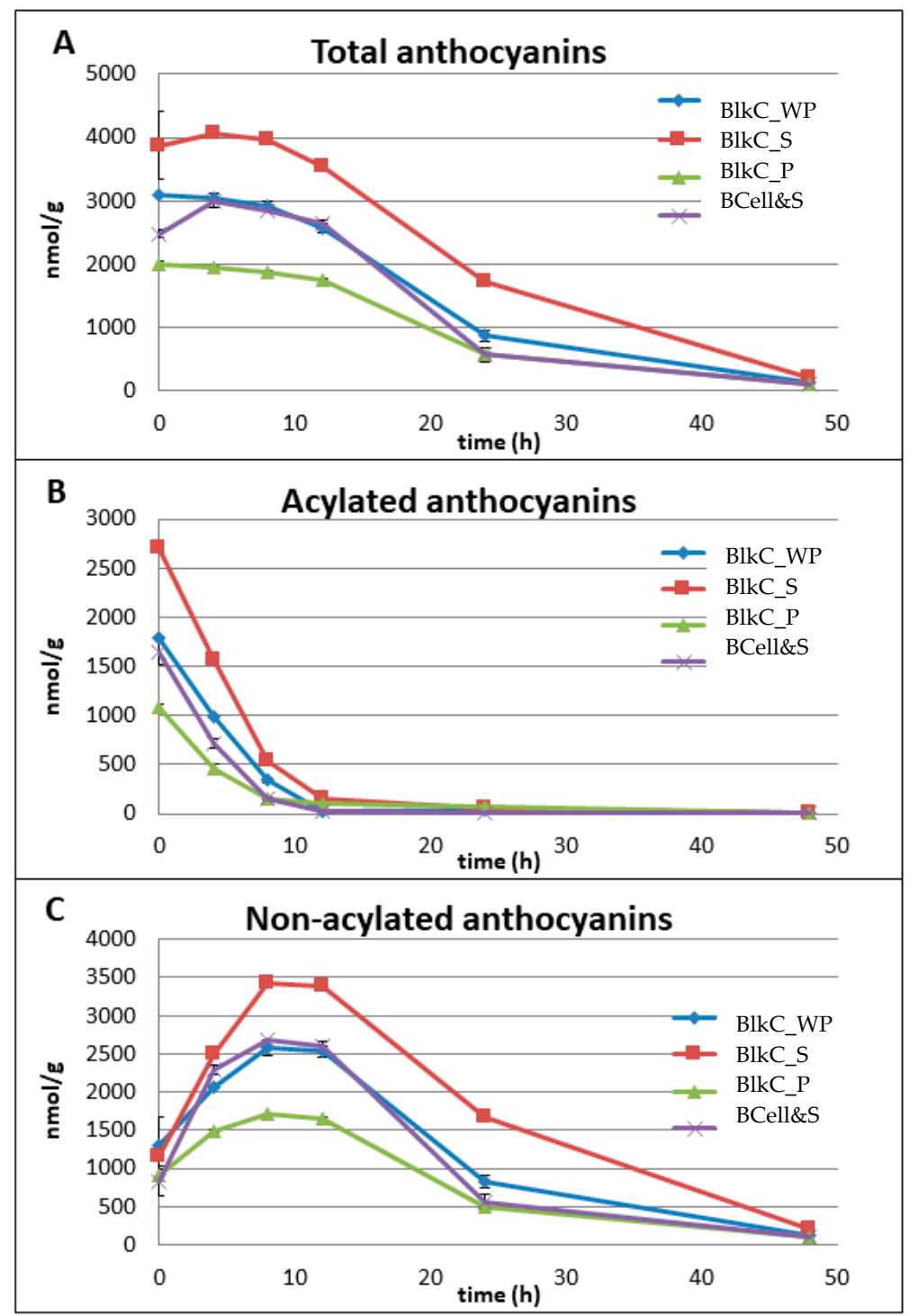

Figure 2. Time course plots for anthocyanins and metabolites for all substrates (time 0 to $48 \mathrm{~h}$ ); data are means $\pm \mathrm{SD}(n=3)$; (A) total anthocyanins (sum of acylated and non-acylated anthocyanins), (B) acylated anthocyanins (sum of caffeic acid derivative of cy-3-O-xylglcgal, sinapic acid derivative of cy-3-O-xylglcgal, ferulic acid derivative of cy-3-O-xylglcgal and p-coumaric acid derivative of cy-3-O-xylglcgal), (C) non-acylated anthocyanins (sum of cy-3-O-xylglcgal and cy-3-O-xylgal). The released (free) acylated and non-acylated anthocyanins were analysed by HPLC-PDA and ESI-LC/MS in the post-fermentation supernatants which were collected at each sampling time $[11,17,32]$. 
Figure $2 \mathrm{~B}$ shows the degradation profiles of acylated anthocyanins, indicating a substantial decrease within the first $8 \mathrm{~h}$ of fermentation. It seems that the pig inoculum in the present study had comparable metabolic activity to a human faecal suspension used in another in vitro fermentation study [28]. In that study, red radish was used as a source of acylated anthocyanins (p-coumaric, ferulic, caffeic and malonic acid derivatives of pg-3-O-sophoroside-5-O-glucoside). A complete degradation of the acylated red radish anthocyanins via two intermediates (pg-3-O-sophoroside-5-O-glucoside and pg-3-O-sophoroside) to 4-hydroxybenzoic acid as well as the hydroxycinnamates p-coumaric, ferulic and caffeic acid was reported [28], though pg-3-O-sophoroside-5-O-glucoside as the corresponding non-acylated anthocyanin (intermediate) was still detectable at $24 \mathrm{~h}$. A similar degradation pattern was seen in the present study: a substantial decrease of acylated anthocyanins within the first $8 \mathrm{~h}$ of fermentation resulted in an almost simultaneous increase of the (corresponding) non-acylated anthocyanins (Figure 2B,C). This increase was likely caused by the generation of cy-3-O-xylglcgal due to the deacylation activity of the microbial inoculum (Supplementary Materials Figure S1). The total amount of acylated cy-3-O-xylglcgal in the black carrot substrates prior to fermentation ranged from 8.5 (BlkC_WP) to $23.1 \mu \mathrm{mol} / \mathrm{g}$ DW (BlkC_S) (Table 4). A "concentration plateau" could be observed between 8 and $12 \mathrm{~h}$, followed by a steady decrease of non-acylated anthocyanins until the end of the fermentation (Figure 2C).

However, it should be noted that low amounts of acylated anthocyanins $(<5 \mathrm{nmol} / \mathrm{g} \mathrm{DM})$ were still detectable at $48 \mathrm{~h}$ of fermentation (Figure 2B). Correa-Betanzo and colleagues also reported low amounts of acetylated anthocyanins after $12 \mathrm{~h}$ of fermentation of a crude blueberry extract [50], using human faeces.

The microbial metabolism of non-acylated anthocyanins has been demonstrated previously using a porcine large intestinal inoculum, [51,52], and human faeces [28,45,53]. Despite differences between both in vitro methodologies in terms of the inoculum (i.e., pig or human; inoculum/sample-ratio), incubation time (e.g., 24 or 72 h), and substrates tested (e.g., standard compounds, red wine/grape juice, encapsulated anthocyanins), a similar metabolic pattern was observed in all studies: i.e., an almost complete "disappearance" of all anthocyanins (glycosides) during fermentation most likely from deglycosylation, as well as cleavage of the anthocyanin heterocyclic ring system, resulting in formation of phenolic acids, such as protocatechuic (from cy-3-O-glucoside), syringic (from mv-3-O-glucoside), and vanillic acids (from pn-3-O-glucoside). A significant decrease of non-acylated anthocyanins from $12 \mathrm{~h}$ to the end of the fermentation period could be seen in the present study, with up to $200 \mathrm{nmol} / \mathrm{g}$ DW (BlkC_S) still detectable at $48 \mathrm{~h}$. Since the analytical procedure was focused on detecting the common black carrot anthocyanins and phenolic acids as well as their degradation profiles, no other phenolic metabolites such as protocatechuic acid were analysed.

In addition to the common acylated and non-acylated black carrot anthocyanins, two other compounds with an intact anthocyanin skeleton could be detected for all substrates during the fermentation process, with concentrations ranging from 128 (BCell\&S) to 257 (BlkC_S) nmol/g DW at $48 \mathrm{~h}$. Based on the UV-Vis spectra and mass-spectrometric data (Figure S2) Compound 1 could be tentatively identified as a pg-based glycoside and Compound 2 as a pn-based glycoside. However, neither of these anthocyanin glycosides could be detected at the start of the fermentation. It was demonstrated by Keppler and Humpf that the O-demethylation activity of (pig) caecal microbiota is in contrast to the well-known O-methylation activity of intestinal and hepatic tissues [52]. However, peonidin is a common methylated metabolite of cyanidin. Furthermore, no reports could be found describing the conversion of cy-glycosides to pg-glycosides during in vitro faecal fermentation. It should also be noted that acylated and non-acylated pg- and pn-glycosides were identified as native constituents in some black carrot cultivars [36]. These findings should therefore be interpreted with caution and further investigations are necessary to clarify the origin of these anthocyanin compounds. 


\subsubsection{Phenolic Acids}

Figure 3 shows the time-concentration degradation profiles of total phenolic acids (A), and the chlorogenic acids (B), as well as the anthocyanin conjugated hydroxycinnamates (C).

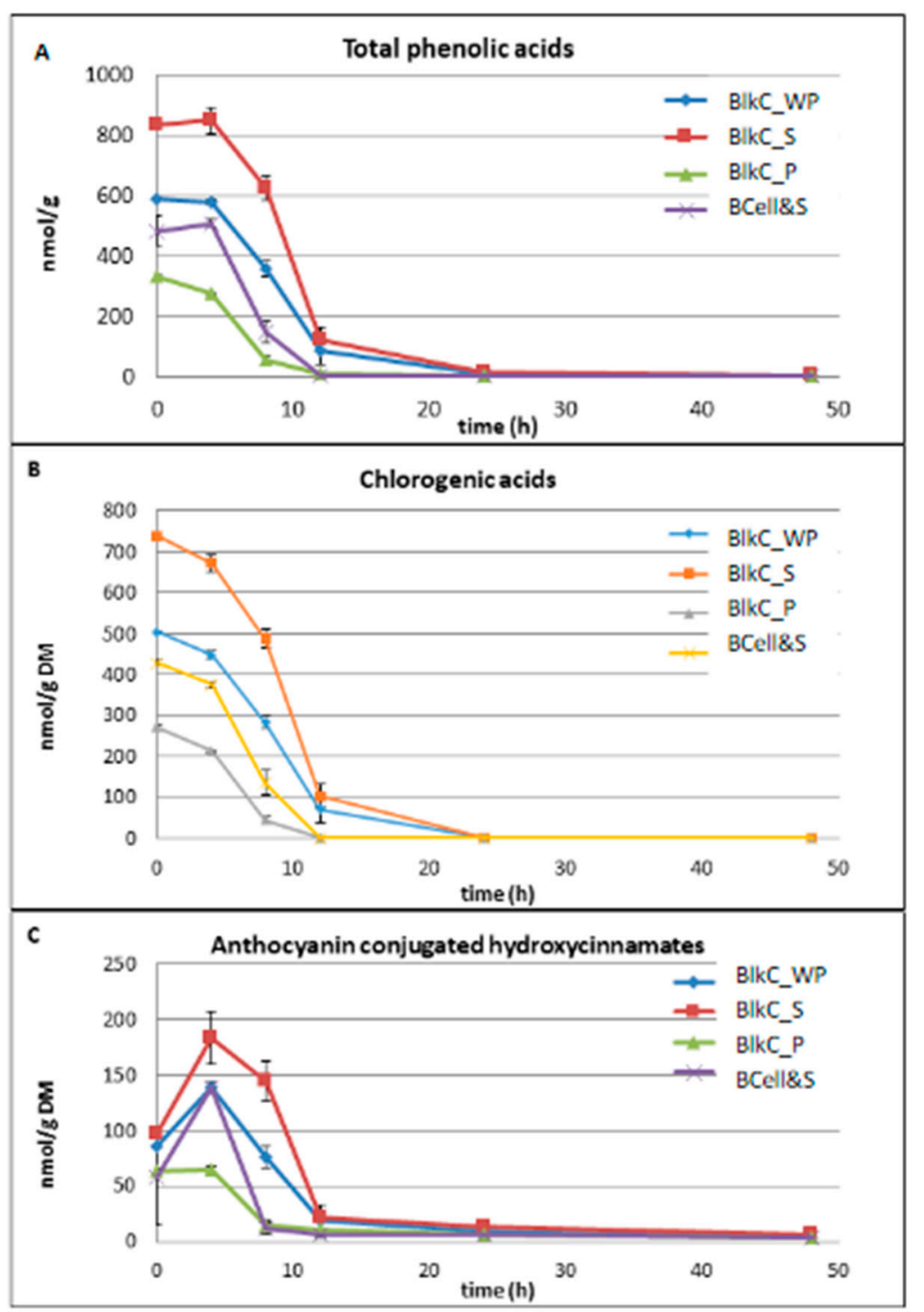

Figure 3. Time course plots for phenolic acids for all substrates (time 0 to $48 \mathrm{~h}$ ); data shown are means $\pm \mathrm{SD}(n=3)$; (A) total phenolic acids (sum of 5-caffeoylquinic (chlorogenic), 3-caffeoylquinic (neochlorogenic), ferulic, sinapic, caffeic, and p-coumaric acid), (B) chlorogenic acids (sum of 5-O-caffeoylquinic, and 3-O-caffeoylquinic acid), and (C) anthocyanin conjugated hydroxycinnamates (sum of caffeic, sinapic, ferulic, and p-coumaric acid).

The concentrations of free caffeic, ferulic, p-coumaric and sinapic acid, usually bound to cy-3-O-xylglcgal to form the acylated anthocyanin fraction in black carrots, reached a maximum at $4 \mathrm{~h}$ of fermentation for all substrates (Figure $3 \mathrm{C}$ ). This was followed by a substantial degradation within the next $4 \mathrm{~h}$ (BlkC_P and BCell\&S) and $6 \mathrm{~h}$ (BlkC_S and BlkC_WP), respectively. Low concentrations $(<5.9 \mathrm{nmol} / \mathrm{g} \mathrm{DW})$ of these four hydroxycinnamic acids could still be measured at the end of the fermentation (Figure 3C). The increases in caffeic, ferulic, p-coumaric and sinapic acid within the first $4 \mathrm{~h}$ of fermentation were most likely a result of the observed degradation (deacylation) of the acylated anthocyanins to their corresponding non-acylated counterparts (Figure 2C). 
The time-concentration degradation profiles of chlorogenic acids were similar for all substrates showing a steady and substantial decrease within the first $12 \mathrm{~h}$ of fermentation (Figure 3B). Neither 5-O-caffeoylquinic (chlorogenic) nor 3-O-caffeoylquinic (neochlorogenic) acid was detectable after $24 \mathrm{~h}$ of fermentation. These findings are in agreement with earlier reports using a human faecal inoculum [54], which showed an intensive metabolism of caffeic acid and chlorogenic acid with no acids detectable after $2 \mathrm{~h}$. The authors identified 3-hydroxyphenylpropionic acid and benzoic acid as the main microbial metabolites of the fermented hydroxycinnamic acids with a similar metabolic pattern for caffeic acid and chlorogenic acid. However, dihydrocaffeic acid, dihydroferulic acid and another unknown compound were identified as the main metabolites in a batch culture fermentation investigating the impact of coffee and chlorogenic acids on the growth of the human gut microbiota [55]. It has been postulated that there is a "funnelled" metabolic pathway for structurally different phenolic acids (and flavonoids) present in tea and/or red wine/grape juice such as caffeic and p-coumaric acids, in which they are first metabolised to intermediates such as 3,4-dihydroxyphenylpropionic acid and 4-hydroxyphenylpropionic acid and then further to 3-phenylpropionic acid as a metabolic "end product" of the microbial fermentation process [45]. However, the authors also noted that the metabolite profiles varied considerably among the 10 individuals studied, and as a consequence health benefits linked to microbial polyphenol metabolites cannot be generalised.

The time-concentration degradation profiles of bacterial cellulose soaked in black carrot supernatant (BCell\&S) and black carrot whole puree (BlkC_WP) were almost identical for acylated and non-acylated anthocyanins, with corresponding total anthocyanins (Figure 2A-C). Similar profiles could be observed for chlorogenic acids (as the main phenolic acids in black carrots), non-esterified hydroxycinnamic acids and total phenolic acids (Figure 3A-C). These findings demonstrate that soaking bacterial cellulose in the "juice" of a fruit or vegetable, may be an appropriate model to examine the fermentability of attached polyphenolic and phenolic compounds.

In 2005, Keppler and Humpf introduced the idea of using the pig as a model system to mimic human microbial polyphenol-metabolism for anthocyanins using commercially available standards [52]. This was followed by work on red wine anthocyanins [51] and flavonol glycosides [56]. Having access to a standardised in vitro model to investigate the microbial metabolism of (controlled) structurally different (poly)phenolics in their natural plant (food) matrix would help to better understand how these compounds are altered by the GIT microbiota. Further in vitro and in vivo studies would be helpful to elucidate the mode of action of black carrots in particular. However, this methodology using an in vitro fermentation technique, and a standard bacterial cellulose combined with supernatants from other fruits and vegetables will be helpful in clarifying how anthocyanins and phenolic acids attached to the PCW, are metabolised by the gut microbiota.

\section{Conclusions}

In vitro fermentations of three fractions of black carrots-the whole puree, the supernatant (juice), and pellet following centrifugation, were investigated. The results showed the effects of the food matrix on disappearance and/or metabolism of anthocyanins (acylated and non-acylated) and phenolic acids, particularly of those polyphenols associated with plant cells (either attached, or trapped within the cell).

Supplementary Materials: The following are available online at http://www.mdpi.com/2304-8158/9/12/1911/s1. Figure S1: Time course plots for cy-3-O-xylglcgal for all substrates (time 0-48 h). Data are means $\pm S D(n=3)$, Figure S2: Representative UPLC chromatogram of BlkC-WP after $48 \mathrm{~h}$. Detection was performed by UPLC-PDA at 520nm and peak identification was carried out by Orbitrap LCMS [11]. Caffeic acid and p-coumaric acid derivatives of cy-3-O-xylglcgal were not detectable after $48 \mathrm{~h}$. 
Author Contributions: Conceptualization, B.A.W. and D.M.; methodology, G.N., D.M., B.M.F. and B.A.W.; software, G.N., D.M. and B.A.W.; validation, G.N., D.M., B.M.F. and B.A.W.; formal analysis, G.N., D.M., B.M.F. and B.A.W.; investigation, G.N., D.M., B.M.F. and B.A.W.; resources, M.J.G.; data curation, G.N., D.M. and B.A.W.; writing - original draft preparation, B.A.W. and D.M.; writing—review and editing, G.N., B.M.F., M.E.N. and M.J.G.; visualization, G.N., D.M. and B.A.W.; supervision, M.J.G.; project administration, M.J.G.; funding acquisition, M.J.G. All authors have read and agreed to the published version of the manuscript.

Funding: This work was funded by the Australian Research Council Centre of Excellence in Plant Cell Walls, grant number CE110010007.

Acknowledgments: Our thanks to Anh Dao Phan, Barbara Gorham Dongjie Wang, Elizabeth McCourt, and Esther Lau for technical assistance, and the Centre for Advanced Imaging at The University of Queensland for access to their facilities.

Conflicts of Interest: The authors report no conflict of interest.

$\begin{array}{ll}\text { Abbreviations } & \\ \text { BlkC } & \text { Black carrot } \\ \text { BCell } & \text { Bacterial cellulose } \\ \text { BCell\&S } & \text { Bacterial cellulose soaked in supernatant } \\ \text { BlkC_P } & \text { Black carrot pellet } \\ \text { BlkC_S } & \text { Black carrot supernatant } \\ \text { BlkC_WP } & \text { Black carrot whole puree } \\ \text { cy } & \text { cyanidin } \\ \text { cy-3-O-xylglcgal } & \text { cyanidin-3-O-xylosyl(glucosyl)galactoside } \\ \text { cy-3-O-xylgal } & \text { cyanidin-3-O-xylosylgalactoside } \\ \text { DM } & \text { Dry matter } \\ \text { GIT } & \text { Gastro-intestinal tract } \\ \text { h } & \text { Hour(s) } \\ \text { min } & \text { Minutes } \\ \text { mv } & \text { malvidin } \\ \text { PC } & \text { Plant cell } \\ \text { PCW } & \text { Plant cell walls } \\ \text { pg } & \text { pelargonidin } \\ \text { pn } & \text { peonidin } \\ \text { SCFA } & \text { Short-chain fatty acids } \\ \end{array}$

\section{References}

1. Costa, C.; Tsatsakis, A.; Mamoulakis, C.; Teodoro, M.; Briguglio, G.; Caruso, E.; Tsoukalas, D.; Margina, D.; Dardiotis, E.; Kouretas, D.; et al. Current evidence on the effect of dietary polyphenols intake on chronic diseases. Food Chem. Toxicol. 2017, 110, 286-299. [CrossRef]

2. Del Rio, D.; Rodriguez-Mateos, A.; Spencer, J.P.E.; Tognolini, M.; Borges, G.; Crozier, A. Dietary (poly)phenolics in human health: Structures, bioavailability, and evidence of protective effects against chronic diseases. Antioxid. Redox Signal. 2013, 18, 1818-1892. [CrossRef]

3. Gonzalez, I.; Morales, M.A.; Rojas, A. Polyphenols and AGEs/RAGE axis. Trends and challenges. Food Res. Int. 2020, 129, e108843. [CrossRef] [PubMed]

4. Pojer, E.; Mattivi, F.; Johnson, D.; Stockley, C.S. The case for anthocyanin consumption to promote human health: A review. Compr. Rev. Food Sci. Food Saf. 2013, 12, 483-508. [CrossRef]

5. Afrin, S.; Giampieri, F.; Gasparrini, M.; Forbes-Hernandez, T.Y.; Cianciosi, D.; Reboredo-Rodriguez, P.; Zhang, J.J.; Manna, P.P.; Daglia, M.; Atanasov, A.G.; et al. Dietary phytochemicals in colorectal cancer prevention and treatment: A focus on the molecular mechanisms involved. Biotechnol. Adv. 2020, 38, e107322. [CrossRef]

6. Pandey, K.B.; Rizvi, S.I. Current understanding of dietary polyphenols and their role in health and disease. Curr. Nutr. Food Sci. 2009, 5, 249-263. [CrossRef] 
7. Santhakumar, A.B.; Kundur, A.R.; Sabapathy, S.; Stanley, R.; Singh, I. The potential of anthocyanin-rich Queen Garnet plum juice supplementation in alleviating thrombotic risk under induced oxidative stress conditions. J. Funct. Foods 2015, 14, 747-757. [CrossRef]

8. Wallace, T.C.; Murray, R.; Zelman, K.M. The nutritional value and health benefits of chickpeas and hummus. Nutrients 2016, 8, e766. [CrossRef]

9. Zhang, D.; Du, M.Z.; Wei, Y.; Wang, C.T.; Shen, L.Q. A review on the structure-activity relationship of dietary flavonoids for protecting vascular endothelial function: Current understanding and future issues. J. Food Biochem. 2018, 42, e12557. [CrossRef]

10. Padayachee, A.; Day, L.; Howell, K.; Gidley, M.J. Complexity and health functionality of plant cell wall fibers from fruits and vegetables. Crit. Rev. Food Sci. Nutr. 2017, 57, 59-81. [CrossRef]

11. Padayachee, A.; Netzel, G.; Netzel, M.; Day, L.; Zabaras, D.; Mikkelsen, D.; Gidley, M.J. Binding of polyphenols to plant cell wall analogues-Part 2: Phenolic acids. Food Chem. 2012, 135, 2287-2292. [CrossRef]

12. Dufour, C.; Loonis, M.; Delosiere, M.; Buffiere, C.; Hafnaoui, N.; Sante-Lhoutellier, V.; Remond, D. The matrix of fruit \& vegetables modulates the gastrointestinal bioaccessibility of polyphenols and their impact on dietary protein digestibility. Food Chem. 2018, 240, 314-322.

13. Hedren, E.; Diaz, V.; Svanberg, U. Estimation of carotenoid accessibility from carrots determined by an in vitro digestion method. Eur. J. Clin. Nutr. 2002, 56, 425-430. [CrossRef]

14. Wink, M. Compartmentation of secondary metabolites and xenobiotics in plant vacuoles. In Advances in Botanical Research Incorporating Advances in Plant Pathology, Vol 25: The Plant Vacuole; Leigh, R.A., Sanders, D., Eds.; Academic Press: Cambridge, MA, USA, 1997; Volume 25, pp. 141-169.

15. Le Bourvellec, C.; Guyot, S.; Renard, C. Non-covalent interaction between procyanidins and apple cell wall material Part I. Effect of some environmental parameters. Biochim. Biophys. Acta Gen. Subj. 2004, 1672, 192-202. [CrossRef]

16. Le Bourvellec, C.; Renard, C. Non-covalent interaction between procyanidins and apple cell wall material. Part II: Quantification and impact of cell wall drying. Biochim. Biophys. Acta Gen. Subj. 2005, 1725, 1-9. [CrossRef]

17. Padayachee, A.; Netzel, G.; Netzel, M.; Day, L.; Zabaras, D.; Mikkelsen, D.; Gidley, M. Binding of polyphenols to plant cell wall analogues-Part 1: Anthocyanins. Food Chem. 2012, 134, 155-161. [CrossRef]

18. Palafox-Carlos, H.; Ayala-Zavala, J.F.; Gonzalez-Aguilar, G.A. The role of dietary fiber in the bioaccessibility and bioavailability of fruit and vegetable antioxidants. J. Food Sci. 2011, 76, R6-R15. [CrossRef]

19. Phan, A.D.T.; Flanagan, B.M.; D'Arcy, B.R.; Gidley, M.J. Binding selectivity of dietary polyphenols to different plant cell wall components: Quantification and mechanism. Food Chem. 2017, 233, 216-227. [CrossRef]

20. Renard, C.M.G.C.; Baron, A.; Guyot, S.; Drilleau, J.F. Interactions between apple cell walls and native apple polyphenols: Quantification and some consequences. Int. J. Biol. Macromol. 2001, 29, 115-125. [CrossRef]

21. Williamson, G.; Clifford, M.N. Colonic metabolites of berry polyphenols: The missing link to biological activity? Br. J. Nutr. 2010, 104, S48-S66. [CrossRef]

22. Bresciani, L.; Favari, C.; Calani, L.; Francinelli, V.; Riva, A.; Petrangolini, G.; Allegrini, P.; Mena, P.; Del Rio, D. The effect of formulation of curcuminoids on their metabolism by human colonic microbiota. Molecules 2020, 25, 940. [CrossRef] [PubMed]

23. Desjardins, Y. Plant polyphenols bioavailability and modulation of the gut microbiota consortium: A paradigm shift in understanding their effects on diseases. In XXIX International Horticultural Congress on Horticulture: Sustaining Lives, Livelihoods and Landscapes; O'Hare, T.J., Netzel, M.E., Eds.; International Society for Horticultural Science (ISHS): Leuven, Belgium, 2015; Volume 1106, pp. 199-210.

24. Cardona, F.; Andres-Lacueva, C.; Tulipani, S.; Tinahones, F.J.; Queipo-Ortuno, M.I. Benefits of polyphenols on gut microbiota and implications in human health. J. Nutr. Biochem. 2013, 24, 1415-1422. [CrossRef] [PubMed]

25. Wang, D.J.; Williams, B.A.; Ferruzzi, M.G.; D'Arcy, B.R. Microbial metabolites, but not other phenolics derived from grape seed phenolic extract, are transported through differentiated Caco-2 cell monolayers. Food Chem. 2013, 138, 1564-1573. [CrossRef] [PubMed]

26. Aura, A.M.; Martin-Lopez, P.; O’Leary, K.A.; Williamson, G.; Oksman-Caldentey, K.M.; Poutanen, K.; Santos-Buelga, C. In vitro metabolism of anthocyanins by human gut microflora. Eur. J. Nutr. 2005, 44, 133-142. [CrossRef]

27. Chalet, C.; Rubbens, J.; Tack, J.; Duchateau, G.S.; Augustijns, P. Intestinal disposition of quercetin and its phase-II metabolites after oral administration in healthy volunteers. J. Pharm. Pharmacol. 2018, 70, 1002-1008. [CrossRef] 
28. Fleschhut, J.; Kratzer, F.; Rechkemmer, G.; Kulling, S.E. Stability and biotransformation of various dietary anthocyanins in vitro. Eur. J. Nutr. 2006, 45, 7-18. [CrossRef]

29. Martini, D.; Del Bo, C.; Porrini, M.; Ciappellano, S.; Riso, P. Role of polyphenols and polyphenol-rich foods in the modulation of PON1 activity and expression. J. Nutr. Biochem. 2017, 48, 1-8. [CrossRef]

30. Rechner, A.R.; Smith, M.A.; Kuhnle, G.; Gibson, G.R.; Debnam, E.S.; Srai, S.K.S.; Moore, K.P.; Rice-Evans, C.A. Colonic metabolism of dietary polyphenols: Influence of structure on microbial fermentation products. Free Radic. Biol. Med. 2004, 36, 212-225. [CrossRef]

31. van Duynhoven, J.; Vaughan, E.E.; Jacobs, D.M.; Kemperman, R.A.; van Velzen, E.J.J.; Gross, G.; Roger, L.C.; Possemiers, S.; Smilde, A.K.; Doré, J.; et al. Metabolic fate of polyphenols in the human superorganism. Proc. Natl. Acad. Sci. USA 2011, 108, 4531-4538. [CrossRef]

32. Phan, A.D.T.; Williams, B.A.; Netzel, G.; Mikkelsen, D.; D'Arcy, B.R.; Gidley, M.J. Independent fermentation and metabolism of dietary polyphenols associated with a plant cell wall model. Food Funct. 2020, 11, 2218-2230. [CrossRef]

33. Mikkelsen, D.; Lopez-Sanchez, P.; Wang, D.; Gidley, M.J. Formation of cellulose-based composites with hemicelluloses and pectins using Komagataeibacter fermentation. In The Plant Cell Wall: Methods and Protocols; Popper, Z.A., Ed.; Humana Press: New York, NY, USA, 2020; Volume 2149, pp. 73-87.

34. Arscott, S.A.; Tanumihardjo, S.A. Carrots of many colors provide basic nutrition and bioavailable phytochemicals acting as a functional food. Compr. Rev. Food Sci. Food Saf. 2010, 9, 223-239. [CrossRef]

35. Gras, C.C.; Carle, R.; Schweiggert, R.M. Determination of anthocyanins from black carrots by UHPLC-PDA after ultrasound-assisted extraction. J. Food Compos. Anal. 2015, 44, 170-177. [CrossRef]

36. Montilla, E.C.; Arzaba, M.R.; Hillebrand, S.; Winterhalter, P. Anthocyanin composition of black carrot (Daucus carota ssp. sativus var. atrorubens Alef.) cultivars Antonina, Beta Sweet, Deep Purple, and Purple Haze. J. Agric. Food Chem. 2011, 59, 3385-3390. [CrossRef] [PubMed]

37. Sun, T.; Simon, P.W.; Tanumihardjo, S.A. Antioxidant phytochemicals and antioxidant capacity of biofortified carrots (Daucus carota L.) of various colors. J. Agric. Food Chem. 2009, 57, 4142-4147. [CrossRef] [PubMed]

38. Zhang, H.; Hassan, Y.I.; Renaud, J.; Liu, R.H.; Yang, C.; Sun, Y.; Tsao, R. Bioaccessibility, bioavailability, and anti-inflammatory effects of anthocyanins from purple root vegetables using mono- and co-culture cell models. Mol. Nutr. Food Res. 2017, 61, 1600928. [CrossRef] [PubMed]

39. Kamiloglu, S.; Ozkan, G.; Isik, H.; Horoz, O.; Van Camp, J.; Capanoglu, E. Black carrot pomace as a source of polyphenols for enhancing the nutritional value of cake: An in vitro digestion study with a standardized static model. LWT Food Sci. Technol. 2016, 77, 475-481. [CrossRef]

40. Padayachee, A.; Netzel, G.; Netzel, M.; Day, L.; Mikkelsen, D.; Gidley, M.J. Lack of release of bound anthocyanins and phenolic acids from carrot plant cell walls and model composites during simulated gastric and small intestinal digestion. Food Funct. 2013, 4, 906-916. [CrossRef] [PubMed]

41. Hestrin, S.; Schramm, M. Synthesis of cellulose by acetobacter-xylinum 2. Preparation of freeze-dried cells capable of polymerizing glucose to cellulose. Biochem. J. 1954, 58, 345-352. [CrossRef]

42. Williams, B.A.; Bosch, M.W.; Boer, H.; Verstegen, M.W.A.; Tamminga, S. An in vitro batch culture method to assess potential fermentability of feed ingredients for monogastric diets. Anim. Feed Sci. Technol. 2005, 123, 445-462. [CrossRef]

43. Williams, B.A.; Mikkelsen, D.; le Paih, L.; Gidley, M.J. In vitro fermentation kinetics and end-products of cereal arabinoxylans and (1,3;1,4)-beta-glucans by porcine faeces. J. Cereal Sci. 2011, 53, 53-58. [CrossRef]

44. Baethgen, W.E.; Alley, M.M. A manual colorimetric procedure for measuring ammonium nitrogen in the soil and plant kjeldahl digests. Commun. Soil Sci. Plant Anal. 1989, 20, 961-969. [CrossRef]

45. Gross, G.; Jacobs, D.M.; Peters, S.; Possemiers, S.; van Duynhoven, J.; Vaughan, E.E.; van de Wiele, T. In vitro bioconversion of polyphenols from black tea and red wine/grape juice by human intestinal microbiota displays strong inter-individual variability. J. Agric. Food Chem. 2010, 58, 10236-10246. [CrossRef] [PubMed]

46. Groot, J.C.J.; Cone, J.W.; Williams, B.A.; Debersaques, F.M.A.; Lantinga, E.A. Multiphasic analysis of gas production kinetics for in vitro fermentation of ruminant feeds. Anim. Feed Sci. Technol. 1996, 64, 77-89. [CrossRef]

47. Williams, B.A.; Bosch, M.W.; Awati, A.; Konstantinov, S.R.; Smidt, H.; Akkermans, A.D.L.; Verstegen, M.W.A.; Tamminga, S. In vitro assessment of gastrointestinal tract (GIT) fermentation in pigs: Fermentable substrates and microbial activity. Anim. Res. 2005, 54, 191. [CrossRef] 
48. Kammerer, D.; Carle, R.; Schieber, A. Characterization of phenolic acids in black carrots (Daucus carota ssp sativus var. atrorubens Alef.) by high-performance liquid chromatography/electrospray ionization mass spectrometry. Rapid Commun. Mass Spectrom. 2004, 18, 1331-1340. [CrossRef]

49. Alasalvar, C.; Grigor, J.M.; Zhang, D.L.; Quantick, P.C.; Shahidi, F. Comparison of volatiles, phenolics, sugars, antioxidant vitamins, and sensory quality of different colored carrot varieties. J. Agric. Food Chem. 2001, 49, 1410-1416. [CrossRef]

50. Correa-Betanzo, J.; Allen-Vercoe, E.; McDonald, J.; Schroeter, K.; Corredig, M.; Paliyath, G. Stability and biological activity of wild blueberry (Vaccinium angustifolium) polyphenols during simulated in vitro gastrointestinal digestion. Food Chem. 2014, 165, 522-531. [CrossRef]

51. Forester, S.C.; Waterhouse, A.L. Identification and toxicity of microfloral anthocyanin metabolites. Am. J. Enol. Vitic. 2008, 59, 351A.

52. Keppler, K.; Humpf, H.U. Metabolism of anthocyanins and their phenolic degradation products by the intestinal microflora. Bioorganic Med. Chem. 2005, 13, 5195-5205. [CrossRef]

53. Flores, G.; del Castillo, M.L.R.; Costabile, A.; Klee, A.; Guergoletto, K.B.; Gibson, G.R. In vitro fermentation of anthocyanins encapsulated with cyclodextrins: Release, metabolism and influence on gut microbiota growth. J. Funct. Foods 2015, 16, 50-57. [CrossRef]

54. Gonthier, M.P.; Remesy, C.; Scalbert, A.; Cheynier, V.; Souquet, J.M.; Poutanen, K.; Aura, A.M. Microbial metabolism of caffeic acid and its esters chlorogenic and caftaric acids by human faecal microbiota in vitro. Biomed. Pharmacother. 2006, 60, 536-540. [CrossRef] [PubMed]

55. Mills, C.E.; Tzounis, X.; Oruna-Concha, M.J.; Mottram, D.S.; Gibson, G.R.; Spencer, J.P.E. In vitro colonic metabolism of coffee and chlorogenic acid results in selective changes in human faecal microbiota growth. Br. J. Nutr. 2015, 113, 1220-1227. [CrossRef] [PubMed]

56. Hein, E.M.; Rose, K.; Van't Slot, G.; Friedrich, A.W.; Humpf, H.U. Deconjugation and degradation of flavonol glycosides by pig cecal microbiota characterized by fluorescence in situ hybridization (FISH). J. Agric. Food Chem. 2008, 56, 2281-2290. [CrossRef] [PubMed]

Publisher's Note: MDPI stays neutral with regard to jurisdictional claims in published maps and institutional affiliations.

(C) 2020 by the authors. Licensee MDPI, Basel, Switzerland. This article is an open access article distributed under the terms and conditions of the Creative Commons Attribution (CC BY) license (http://creativecommons.org/licenses/by/4.0/). 